\title{
Patient's Reactions to Digital Rectal Examination of the Prostate
}

\author{
Andre B. Furlan, Rafael Kato, Fabio Vicentini, Jose Cury, Alberto A. Antunes, Miguel Srougi
}

Division of Urology, University of Sao Paulo, USP, Sao Paulo, SP, Brazil

\begin{abstract}
Objective: In recent years, there has been a rise in the incidence of prostate cancer (PCa), and routine screening for the disease has become a well accepted clinical practice. Even with the recognized benefit of this approach, some men are still reluctant to undergo digital rectal examination (DRE). For this reason, we designed the present study in order to better understand men's reactions about this method of screening. The aim was to identify possible drawbacks that could be overcome to increase DRE.

Materials and Methods: We randomly selected 269 patients that were enrolled in an institutional PCa screening program. They were first asked to answer a question regarding their preferred position to undergo the examination. Following this step, they answered a questionnaire in which physical and psychological reactions regarding the DRE were presented. Finally, we used a visual analogical scale (VAS) to analyze the perception of pain during DRE.

Results: The supine position was preferred for most patients (53.9\%). Before DRE, about 59.4\% of patients felt that the exam would be acceptable. After DRE, this figure increased to $91.5 \%(p<0.001)$. Mean VAS score during DRE was 1.69 on a scale with a range between 0 and $10(0=$ no pain; $10=$ extreme pain $)$.

Conclusion: Patient expectations about DRE were negative before examination and changed significantly following the exam. Pain during examination was negligible, contrary to the prevalent belief. These two findings must be clearly presented to patients in order to improve PCa screening acceptance.
\end{abstract}

Key words: prostatic neoplasms; digital rectal examination; diagnosis

Int Braz J Urol. 2008; 34: 572-6

\section{INTRODUCTION}

Despite a certain degree of imprecision, digital rectal examination (DRE) still represents a useful method to identify prostate cancer (PCa) cases $(1,2)$. Among the available diagnostic tools, this method is the fastest, cheapest, and most accessible to patients. However, a great number of men still refuse to undergo DRE (3), with reasons for this behavior varying from lack of knowledge about the disease to cultural prejudice related to the examination (3).

Despite some advantages, DRE has some limitations (4), most of them are related to determina- tion of the prostate volume and the initial detection of PCa cases (5). Furthermore, its sensitivity depends on the expertise of the physician, and if there is a large amount of inter-observer variability.

Community studies analyzing the reason why men refuse to undergo DRE are of pivotal importance to the development of public health strategies aimed at PCa screening. The objectives of the present study are to analyze patients' preferred examination position, to analyze the patients' expectations and reactions regarding DRE before and after the examination, to analyze the level of pain felt during the exam, and finally, to define the acceptance of PCa screening performed annually. 


\section{MATERIALS AND METHODS}

Among 1070 men who participated in a $\mathrm{PCa}$ screening program, $269(25.14 \%)$ were randomly chosen to participate the study. The ages of the patients assessed varied from 45 to 86 years, and all men were interviewed by undergraduate medical students under the supervision of experienced urologists. All patients underwent blood analysis for measurement of prostate specific antigen (PSA), urinalysis, and DRE in the supine position by experienced urologists. Patients also completed questionnaires related to quality of life, urinary symptoms, and sexual function.

Following these steps, patients answered the questions presented in Figure-1. The first question concerned the preferred position for DRE. The options were standing up, kneeling while resting on the elbows, supine, and left lateral positions. The second question concerned the subjects' expectations of how it was going to be. Figure- 2 included 3 questions about patient's reactions after examination. The first question concerned the subjects' expectations on how it was. The second question concerned the acceptance of the annual screening procedure and the third the perception of pain related to the exam on a score from 0 to 10 according to a visual analogical scale (VAS).

The impression of patients regarding the DRE was classified according to the answer about the expectation before and the reactions after DRE. Patients who answered normal or not comfortable were considered to have a good impression about the examination; conversely, those who responded humiliating or painful were considered to have a bad impression about the examination.
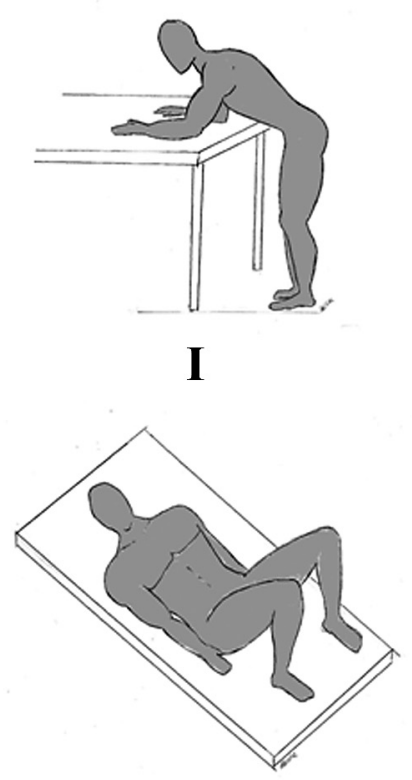

III

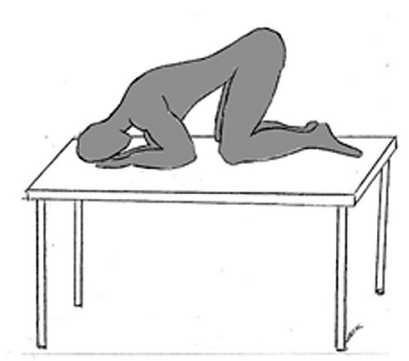

II

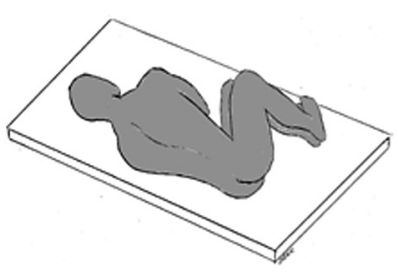

Figure 1

1) In which position would you rather be examined?

2) Before the exam, what was your impression about digital rectal examination?

$\square$ Painful

$\square$ Humiliating

$\square$ Not comfortable

$\square$ Normal 


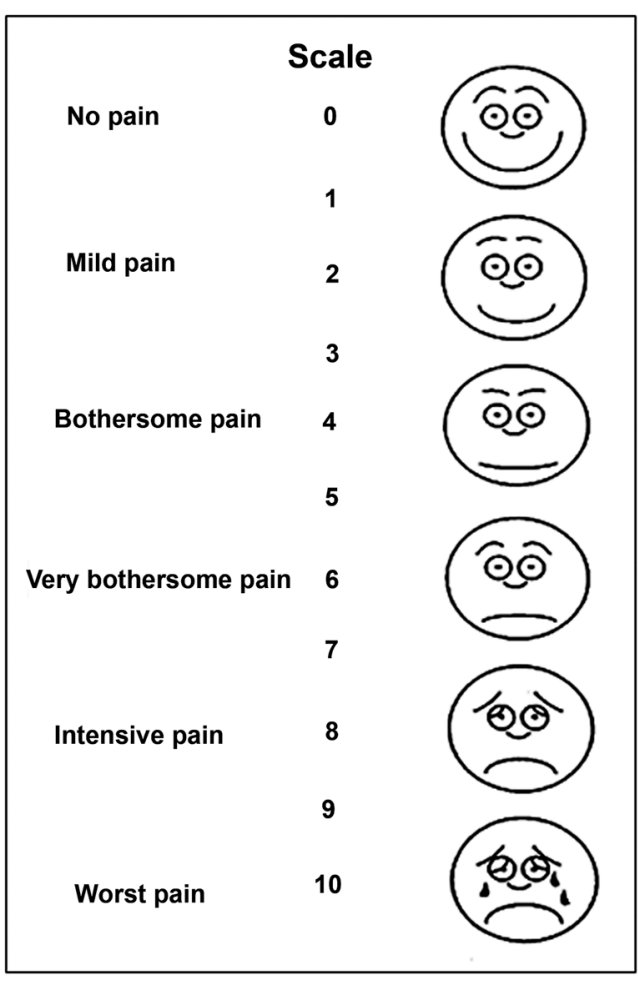

Figure 2

1) After the exam, what is your impression about DRE?

$\square$ Painful

$\square$ Humiliating

$\square$ Not comfortable

$\square$ Normal

2) Would you repeat this exam annually?

$\square$ Yes

$\square$ No

3) How do you classify pain in during this exam?

For statistical analysis we used the McNeman Chi-squared test. Statistical analysis was performed using the SPSS 12.0 for Windows software and significance was set as $\mathrm{p} \leq 0.05$.

\section{RESULTS}

Table-1 shows the results of the patients' answers when questioned about the position they

would choose to undergo DRE. More than half of the patients preferred the supine position, and kneeling while resting on the elbows was the least attractive.

Figure-1 illustrates the patients' expectations about DRE. Notably, before undergoing the exam, $54 \%$ of patients imagined that it would be painful, humiliating, or bothersome. After the exam, 137 $(50.9 \%)$ patients maintained their answer, while 132 (49.1\%) changed their answer and expressed a good impression about the exam. Also, before the exam, 160 of the 269 patients $(59.5 \%)$ imagined that the exam would not cause discomfort. After DRE, 246 out of 269 patients $(91.4 \%)$ had a good impression $(\mathrm{p}<0.001)$ (Figure-2).

When the men were asked if they would be willing to repeat the examination annually, with the aim of screening for PCa, only five patients answered negatively. The other $264(98.1 \%)$ patients said that they would repeat the exam without foreseeing any problem.

The mean pain score related to DRE as reported on the VAS was 1.68 (median 1).

\section{COMMENTS}

The present study analyzed the reactions of men regarding DRE who had never previously undergone this examination. To justify this study, it is necessary to consider that because of their pervasive heterosexual culture, for many decades Latin-Americans have been extremely hesitant to undergo this kind of examination.

Table 1 - Patients' preferred position for digital rectal examination.

\begin{tabular}{lrr}
\hline Position & N & (\%) \\
\hline Standing up & 76 & 28.25 \\
Kneeling while resting & 19 & 7.07 \\
$\quad$ on the elbows & & \\
Supine & 145 & 53.90 \\
Left lateral & 29 & 10.78 \\
Total & 269 & 100 \\
\hline
\end{tabular}


In the post-PSA era, there was a great advance when patients' wives and girlfriends became supportive of the urologists' cause, convincing patients that a man's prostate examination had the same significance as a woman's preventive gynecologic examination. With this example and with a great appeal from the medical community, there was a large amount of support for men to adhere to PCa screening programs. The extent of this cultural revolution could be proven by the fact that in only one day of attending $\mathrm{PCa}$ screening, we found 1070 men who were subsequently included in this study.

Based on the data of the present study, the expectations before the examination showed that half of the men were not worried because they imagined that the examination would be non-traumatic; this expectation was not only confirmed but increased after DRE.

One hundred nine patients did not have a favorable previous impression about the examination, but after the exam, only 23 men maintained this impression. It is important to point out that the level of pain reported when they underwent the exam was extremely low, 1.69 on a scale from 0 to 10 , demonstrating numerically what specialists have been repeating continuously to patients.

In the medical literature, we found only one reference in which the authors compared two methods of prostate examination, with better acceptance of the standing position, with the body bending forward and supported by the elbow, than of the left lateral position (5). In the present report, the supine position was the preference of more than half of the patients. The majority of assistant physicians, seniors or juniors, also preferred the supine position, which allows a better impression of the prostate characteristics.
Scientifically unmasking the DRE, as we have shown, supplies important information to the physicians who are dedicated to PCa screening and to public health care problems. Our data clearly demonstrate that DRE is far from being a humiliating or painful exam. These figures indicate that when candidates for PCa screening are properly advised and treated with the humanistic principles that govern good medical practice, almost $100 \%$ of them promise to return annually to undergo the examination.

\section{CONFLICT OF INTEREST}

None declared.

\section{REFERENCES}

1. Srougi M: Cancer da Próstata. In: Srougi M, Simon SD (ed.), Câncer Urológico. São Paulo, Platina. 1996; pp. 281-359.

2. Tenke P, Horti J, Balint P, Kovacs B: Prostate cancer screening. Recent Results Cancer Res. 2007; 175: 6581.

3. Nijs HG, Essink-Bot ML, DeKoning HJ, Kirkels WJ, Schröder FH Why do men refuse or attend populationbased screening for prostate cancer? J Public Health Med. 2000; 22: 312-6.

4. Fournier G, Valeri A, Mangin P, Cussenot O: Prostate cancer: Diagnosis and staging. Ann Urol (Paris). 2004; 38: 207-24.

5. Frank J, Thomas K, Oliver S, Andrews S, Choong S, Taylor R, et al.: Couch or crouch? Examining the prostate: a randomized study comparing the knee-elbow and the left-lateral position. BJU Int. 2001; 87: 331-3. 


\section{EDITORIAL COMMENT}

The authors have analyzed one aspect of digital rectal examination (DRE) that, at first glance only, could be considered insignificant. In fact, we have to consider that the screening program was conducted in a geographic area where the predominant Latin-Americans have a great hesitation to undergo this kind of exam due to cultural and religious reasons. I fully agree that the results of this study should support the urologists and the general physicians to convince the patient that this type of exam is far from being humiliating and painful. If the cultural revolution continues on this course, DRE can be considered in the mind of the general population at the same level of a woman's preventive gynecologic exam.
Unfortunately, many physicians are still reluctant to perform a DRE due to the lack of experience or due to culture reasons. Moreover, it should be remembered that the positive predictive value of DRE is limited as predictor of prostate cancer diagnosis (1), and an effective program of prostate cancer prevention has to be accompanied by a PSA examination.

\section{REFERENCES}

1. Issa MM, Zasada W, Ward K, Hall JA, Petros JA, Ritenour $\mathrm{CW}$, et al.: The value of digital rectal examination as a predictor of prostate cancer diagnosis among United States Veterans referred for prostate biopsy. Cancer Detect Prev. 2006; 30: 269-75.

\author{
Dr. Vincenzo Scattoni \\ Department of Urology \\ University Vita-Salute \\ Scientific Institute San Raffaele \\ Milan, Italy \\ E-mail: scattoni.vincenzo@hsr.it
}

\section{EDITORIAL COMMENT}

Digital rectal examination (DRE) is clearly not an ideal general screening tool for prostate cancer (if one believes that it should be done at all). Nevertheless, proposing DRE to his male patients the urologist raises their awareness of prostate diseases, and provided sufficient explanation is given, it should certainly be included in regular check-ups. The authors should be commended for demystifying DRE.
In addition I feel that this paper is a good illustration of an excellent clinical paper, tackling a single question, designing a well conducted study and providing a straight answer and conclusion.

Dr. Paul J. Van Cangh Division of Urology Cliniques Universitaires St Luc Brussels, Belgium E-mail:paul.vancangh@uclouvain.be 